\title{
ESPECIES ALTERNATIVAS DE Eucalyptus EN LA REGIÓN DEL BIO-BIO RESULTADOS DE CRECIMIENTO A LOS 14 AÑOS DE EDAD
}

\author{
Emhart, Verónica ${ }^{5}$; Celhay, Juan Andrés ${ }^{5}$; Velilla, Edgardo ${ }^{5}$ y Medina, Alex ${ }^{5}$
}

\section{RESUMEN}

La producción comercial de especies de Eucalyptus en Chile está basada principalmente en $E$. globulus ssp globulus y $E$. nitens. Frente a nuevos desafíos de mercado y condiciones medioambientales, como la presencia de nuevas plagas o el cambio climático, es necesario tener otros materiales genéticos disponibles y evaluados en el patrimonio nacional.

El presente estudio se estableció en predios de Forestal Mininco en el año 1997 en suelos trumaos y en rojo arcillosos con riego con la finalidad de evaluar productividad de otras especies de eucalipto alternativas a E. globulus ssp globulus y a E. nitens.

Las especies probadas fueron E. badjensis, E. viminalis, E. smithii, E. cypellocarpa, E. urophylla, E. benthamii, E. camaldulensis, E. cinerea, E. delegatensis, E. globulus ssp globulus, E. globulus ssp pseudoglobulus, E. globulus ssp maidenii, E. nitens, Acacia mearnsii, Acacia dealbata y Casuarina equisetifolia.

Después de 14 años de crecimiento, dentro de las especies con mayor volumen se encuentran E. badjensis con valores cercanos a los 776 y $887 \mathrm{~m}^{3}$, E. viminalis entre 645 y $760 \mathrm{~m}^{3}$, seguido por E. smithii con 542 y $765 \mathrm{~m}^{3}$ para ambas condiciones de sitio.

E. globulus ssp globulus también presenta buenos crecimientos entre 610 a $572 \mathrm{~m}^{3}$, y $E$. nitens alcanza entre 493 y $603 \mathrm{~m}^{3}$ para las 2 condiciones de sitio.

Dado estos resultados y a la edad del ensayo, el siguiente paso será corroborar las características de la madera para proyectar los productos, sean pulpables o sólidos o como biomasa.

Palabras claves: Eucalyptus, productividad, especies alternativas

${ }^{5}$ Forestal Mininco SA, Los Ángeles. Chile. 


\section{SUMMARY}

Eucalypt commercial production in Chile is based on mainly two species, E. globulus ssp globulus y $E$. nitens. To face future market challenges or changes in environmental conditions, like pest and diseases or climate change it is necessary to have other genetic materials available and assessed in the country.

The present study was established in 1997 in Forestal Mininco farms over 2 different soils types; volcanic ashes and red clays with irrigation. The goal is to assess alternative eucalypt species to E. globulus ssp globulus and $E$. nitens productivity.

Tested species included E. badjensis, E. viminalis, E. smithii, E. cypellocarpa, E. urophylla, E. benthamii, E. camaldulensis, E. cinerea, E. delegatensis, E. globulus ssp globulus, E. globulus ssp pseudoglobulus, E. globulus ssp maidenii, E. nitens, Acacia mearnsii, Acacia dealbata and Casuarina equisetifolia.

At 14 years old, species with great volume potential volume are E. badjensis with productivity levels close to 776 and up to $887 \mathrm{~m}^{3}$, E. viminalis from 645 to $760 \mathrm{~m}^{3}$, follow by $E$. smithii with 542 to $765 \mathrm{~m}^{3}$ for both site conditions.

E. globulus ssp globulus and E. nitens shows also a good growth at the two sites, with 610 and $572 \mathrm{~m}^{3}$, and 493 and $603 \mathrm{~m}^{3}$, respectively.

Given these results and the age of assessment, the next step should be to check wood quality properties to project possible products as pulp, solid or biomass.

Keywords: Eucalyptus, productivity, alternative species 


\section{INTRODUCCIÓN}

La producción comercial de especies de Eucalyptus en Chile está basada principalmente en E. globulus ssp globulus y E. nitens. Existen en el país alrededor de 648 mil hectáreas plantadas con estas 2 especies y alrededor de 20 mil hectáreas han sido plantadas con otras especies de Eucalyptus, lo que corresponde al $1 \%$ de la superficie total nacional. El desempeño de E. globulus ssp globulus y $E$. nitens ha sido notable para la industria de la pulpa y las exportaciones en el país.

No obstante, la presencia de plagas en los últimos años, como por ejemplo la llegada y masificación de Gonypterus $s p$, ha generado la necesidad de buscar otras alternativas para la producción de pulpa, también de biomasa y para fijación de carbono.

Frente a nuevos desafíos de mercado y condiciones medioambientales como la presencia de nuevas plagas y el cambio climático es necesario tener otros materiales genéticos disponibles y evaluados en el patrimonio nacional. Es por eso que en los años 70 se introdujeron varias especies de Eucalyptus en el país cuyo objetivo fue evaluar el desempeño de otras especies alternativas a las ya comerciales, como el caso de Pinus radiata. Esto también fue llevado a cabo en patrimonio de CMPC en los años 80 y 90 y en 2011, siendo probadas distintas alternativas tanto para la producción de pulpa, como de biomasa, y resultados parciales de esto son presentados en este artículo.

Existe poca información pública sobre especies alternativas de Eucalyptus que podrían ser potenciales para la Región del Bio Bio. Algunos resultados fueron publicados por INFOR sobre potencial para E. delegantensis con crecimientos interesantes (INFOR, 1986). Existen otras iniciativas particulares de empresas forestales pero que no se encuentran publicadas.

El objetivo de este estudio fue evaluar el crecimiento y la supervivencia de distintas especies de Eucalyptus en dos condiciones de sitio en la Región del Bio Bio, en patrimonio de Forestal Mininco.

De las especies probadas, E. badjensis tiene una distribución natural bastante restringida a una pequeña región en el plateau sur al sureste de Nueva Gales del Sur y ha tenido buen desarrollo en ensayos de especies en Australia por lo que ha generado considerable interés. Los requerimientos climáticos se encuentran en el Cuadro $N^{\circ} 1$ (Jovanovic y Booth, 2002).

E. benthamii tiene una distribución bien restringida en su lugar de origen a una pequeña área al oeste de Sydney. Esta especie ha tenido tasas de crecimiento interesantes en ensayos en Australia y otros países. Los usos de su madera aún no están completamente evaluados, sin embargo, se ve como una especie con gran potencial para producción comercial. Tiene tolerancia al frío y a la sequía. Los requerimientos climáticos se encuentran en Cuadro $\mathrm{N}^{\circ} 1$ (Jovanovic y Booth, 2002).

E. camaldulensis es uno de eucaliptos más plantados en el mundo. Su distribución natural abarca casi toda Australia (Boland et al., 1989) y se la encuentra cerca de cursos de agua lo que que a menudo le permite sobrevivir en regiones áridas o semiáridas. Las condiciones climáticas de las procedencias del sur de Australia se describen el Cuadro $\mathrm{N}^{\circ} 1$. Su madera tiene usos variados, como por ejemplo construcción, durmientes de ferrocarriles, debobinado y pulpa.

E. smithii tiene una distribución restringida en algunas zonas de plateau, en las costas escarpadas adyacentes y las tierras bajas costeras al sur de Nueva Gales del Sur. Su distribución también se extiende a Victoria en la región de Gippsland (Boland et al., 1989). Su madera se utiliza para construcción en general (Jovanovic y Booth, 2002).

E. viminalis se encuentra ampliamente distribuido en el sudeste de Australia, entre las latitudes $28^{\circ}$ y $43^{\circ} \mathrm{S}$. Es común encontrarlo en el este de Tasmania, las islas del Estrecho de Bass y el sur de Victoria. Su rango altitudinal comprende desde nivel del mar hasta los $1.400 \mathrm{msnm}$. 
Presenta tolerancia al frío y su madera ha sido usada para pisos y paneles, así como madera juvenil ha sido usada para producción de pulpa. Las condiciones climáticas donde crece $E$. viminalis se mencionan en el Cuadro $\mathrm{N}^{\circ} 1$.

\section{Cuadro $\mathrm{N}^{\circ} 1$}

CONDICIONES CLIMÁTICOS EN SUS ÁREAS DE ORIGEN DE ALGUNAS DE LAS ESPECIES INCLUIDAS EN EL ENSAYO DE INTRODUCCIÓN DE EUCALIPTOS EN LOS PREDIOS RUCAMANQUI Y MALVÉN

\begin{tabular}{|l|c|c|c|c|c|}
\hline $\begin{array}{l}\text { Especie/ } \\
\text { Característica }\end{array}$ & $\begin{array}{c}E . \\
\text { badjensis }\end{array}$ & $\begin{array}{c}\boldsymbol{E} \text {. } \\
\text { benthamii }\end{array}$ & $\begin{array}{c}\boldsymbol{E} \text {. } \\
\text { camaldulensis }\end{array}$ & $\begin{array}{c}\boldsymbol{E} \\
\text { smithii }\end{array}$ & $\begin{array}{c}\boldsymbol{E} \\
\text { viminalis }\end{array}$ \\
\hline $\begin{array}{l}\text { Precipitación } \\
\text { media anual } \\
\text { (mm) }\end{array}$ & $580-1.230$ & $730-1.010$ & $400-2.000$ & $610-1.930$ & $500-2.500$ \\
\hline $\begin{array}{l}\text { Régimen de } \\
\text { Iluvias }\end{array}$ & $\begin{array}{c}\text { Uniforme/ } \\
\text { bimodal } \\
\text { Verano }\end{array}$ & $\begin{array}{c}\text { Uniforme/ } \\
\text { bimodal } \\
\text { Verano }\end{array}$ & $\begin{array}{c}\text { Uniforme } \\
\text { Invierno }\end{array}$ & $\begin{array}{c}\text { Uniforme } \\
\text { Invierno }\end{array}$ & $\begin{array}{c}\text { Uniforme } \\
\text { Invierno y } \\
\text { verano }\end{array}$ \\
\hline $\begin{array}{l}\text { Período seco } \\
\text { (meses) }\end{array}$ & $0-5$ & $0-5$ & $0-7$ & $0-6$ & $0-5$ \\
\hline $\begin{array}{l}\mathrm{T}^{\circ} \text { máxima } \\
\text { media mes } \\
\text { más cálido } \\
\left({ }^{\circ} \mathrm{C}\right)\end{array}$ & $22-27$ & $26-30$ & $21-41$ & $20-27$ & $21-32$ \\
\hline $\begin{array}{l}\mathrm{T}^{\circ} \text { mínima } \\
\text { media mes } \\
\text { más frío }\left({ }^{\circ} \mathrm{C}\right)\end{array}$ & $-3-3$ & $-1-3$ & $0-14$ & $-3-7$ & $-4-9$ \\
\hline $\begin{array}{l}\mathrm{T}^{\circ} \text { media } \\
\text { anual }\left({ }^{\circ} \mathrm{C}\right)\end{array}$ & $7-15$ & $13-17$ & $10-25$ & $7-17$ & $4-17$ \\
\hline
\end{tabular}

Procedencias del S

\section{MATERIAL Y METODO}

\section{Descripción del Estudio}

En el año 1997 se plantó 2 ensayos en la región de Bio Bio, cuya descripción se detalla en el Cuadro $\mathrm{N}^{\circ}$ 2. Un ensayo fue localizado en la comuna de Tucapel y el segundo en la comuna de Negrete. La superficie de cada uno es de 1,9 ha.

Los sitios donde fueron establecidos los ensayos se caracterizan por tener buenas condiciones para el crecimiento de eucaliptos; suelos de ceniza volcánica en el caso del fundo Rucamanqui y suelos rojos arcillosos en el caso de Malvén, y precipitaciones anuales de alrededor de $1.300 \mathrm{~mm}$, con la mayor parte distribuida en los meses de invierno y un menor porcentaje en el resto del año, aunque pueden presentar periodos secos importantes de 3 a 4 meses.

El sitio de Malvén tuvo riego por surcos en los primeros 7 años de vida, el sitio de Rucamanqui no fue regado.

Los tratamientos incluidos en los ensayos comprenden 13 especies de Eucalyptus, 2 de Acacia y una de Casuarina, como se indica en el Cuadro $N^{\circ} 3$. 
Cuadro $\mathrm{N}^{\circ} 2$

DESCRIPCIÓN DE LOS ENSAYOS DE INTRODUCCIÓN DE ESPECIES PLANTADOS EN 1997

\begin{tabular}{|l|c|c|}
\hline Fundo / Descripción & Rucamanqui & Malvén \\
\hline Año plantación & 1997 & 1997 \\
\hline Suelo & Ceniza volcánica & Rojo arcilloso \\
\hline Régimen hídrico & Sin riego & Con riego \\
\hline Latitud S & $37^{\circ} 10^{\prime} 52^{\prime \prime}$ & $37^{\circ} 43^{\prime} 50^{\prime \prime}$ \\
\hline Longitud W & $71^{\circ} 51^{\prime} 29^{\prime \prime}$ & $72^{\circ} 22^{\prime} 06^{\prime \prime}$ \\
\hline Altitud $(\mathrm{msnm})$ & 470 & 170 \\
\hline Precipitación media anual $(\mathrm{mm})$ & 1.389 & 1.354 \\
\hline Temperatura media anual $\left({ }^{\circ} \mathrm{C}\right)$ & 11,6 & 12,2 \\
\hline Diseño & Bloques Completos al Azar & Bloques Completos al Azar \\
\hline $\mathrm{N}^{\circ}$ tratamientos & 16 & 16 \\
\hline $\mathrm{N}^{\circ}$ árboles/parcela & 81 & 81 \\
\hline $\mathrm{N}^{\circ}$ Réplicas & 3 & 3 \\
\hline Espaciamiento inicial $(\mathrm{m} \times \mathrm{m})$ & $3 \times 1,6$ & $3,2 \times 1,5$ \\
\hline Superficie (ha) & 1,9 & 1,9 \\
\hline
\end{tabular}

Cuadro $\mathrm{N}^{\circ} 3$

DESCRIPCIÓN DE LOS TRATAMIENTOS INVOLUCRADOS EN LOS ENSAYOS FUNDOS RUCAMANQUI Y MALVÉN

\begin{tabular}{|c|c|l|c|c|}
\hline $\begin{array}{c}\text { Trat. } \\
\mathbf{N}^{\circ}\end{array}$ & Genotipo & \multicolumn{1}{|c|}{ Especie } & Origen & Localidad \\
\hline 1 & S9726 & Acacia mearnsii & Australia & Desconocida \\
2 & S9720 & Eucalyptus cypellocarpa & Australia & Desconocida \\
3 & S4726 & Eucalyptus nitens & Australia & Tallaganda SF \\
4 & S9725 & Eucalyptus globulus ssp. pseudoglobulus & Australia & Desconocida \\
5 & S1703 & Eucalyptus globulus ssp. globulus & Australia & Jeeralang \\
6 & S9684 & Acacia dealbata & Australia & Queen Vic. Terrace \\
7 & S9727 & Casuarina equisetifolia & Chile & - \\
8 & S9732 & Eucalyptus badjensis & Australia & Desconocida \\
9 & S9729 & Eucalyptus urophylla & Australia & Desconocida \\
10 & S9730 & Eucalyptus delegatensis & Chile & Octava Región \\
11 & S9731 & Eucalyptus globulus ssp. maidenii & Australia & Desconocida \\
12 & S4829 & Eucalyptus smithii & Australia & Orbost \\
13 & S10055 & Eucalyptus benthamii & Australia & Kedumba,Val. \\
14 & S9779 & Eucalyptus camaldulensis & Australia & Lake Albacutya \\
15 & S4819 & Eucalyptus viminalis & Australia & Bendoc \\
16 & S11006 & Eucalyptus cinerea & Desconocido & Desconocida \\
\hline
\end{tabular}

\section{Mediciones y Análisis de datos}

Los ensayos fueron medidos a fines del 2011, cuando cumplieron 14 años de edad. Las variables consideradas fueron DAP, altura y estado sanitario, con un criterio forestal productivo. La supervivencia fue estimada en función de los árboles vivos a los 14 años como porcentaje de los 
árboles plantados en total para cada tratamiento y réplica.

Debido a que para muchas de las especies no se cuenta con fórmulas para estimación de volumen, se utilizó una fórmula de árbol individual que considera el volumen de un cilindro por un factor de forma para cada especie. Luego el volumen se expandió por número de árboles por hectárea. Finalmente, se calcularon los promedios por ensayo y tratamiento.

\section{RESULTADOS Y DISCUSIÓN}

\section{Ensayo Rucamanqui}

En el sitio de Rucamanqui la supervivencia general del ensayo fue de $72 \%$ (Figura № 1 ). Dentro de las especies de Eucalyptus la supervivencia obtenida alcanza al 74\%. En general, la supervivencia es aceptable para la gran mayoría de las especies, excepto $E$. urophylla que fue de $18 \%$, y en E. smithii y E. camaldulensis que fue de $54 \%$. En general, los eventos de heladas en Rucamanqui afectaron la supervivencia de E. urophylla y E. camaldulensis.

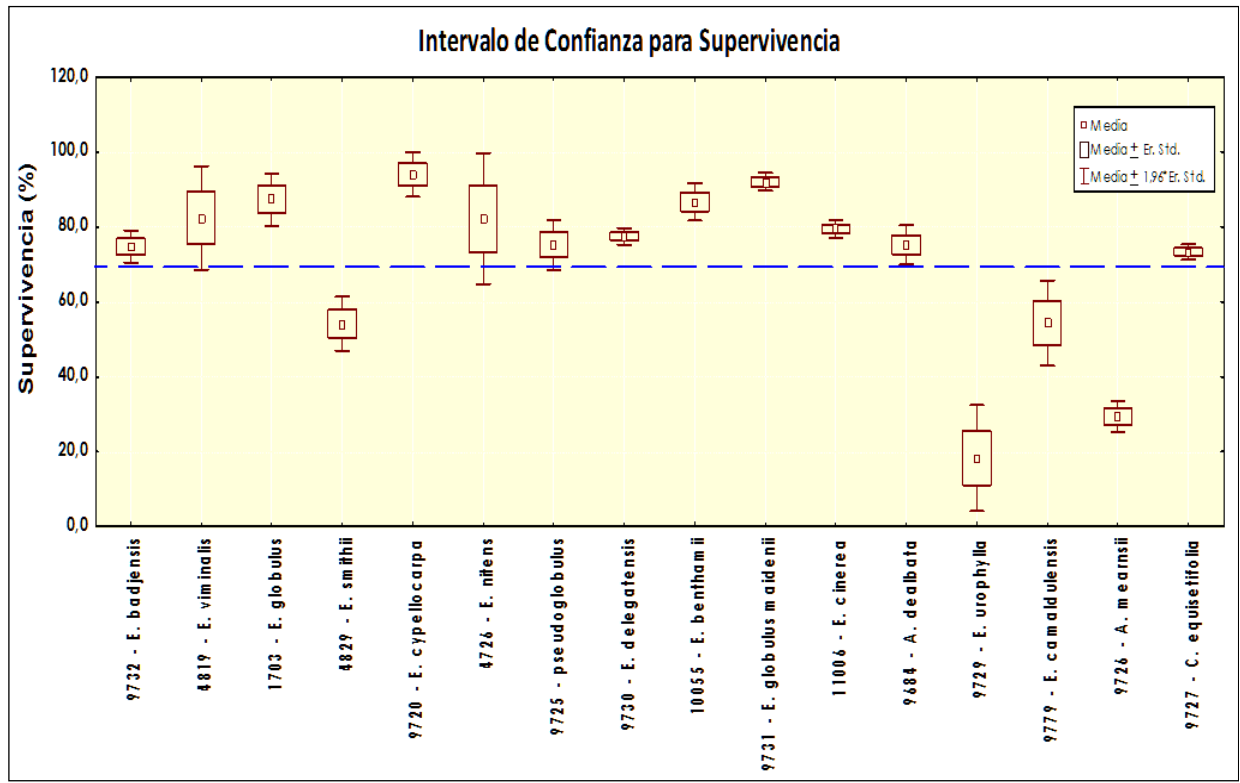

Figura $\mathrm{N}^{\circ}$

SUPERVIVENCIA DE LAS ESPECIES EN ENSAYO RUCAMANQUI 14 AÑOS EDAD

Respecto del crecimiento después de 14 años, el promedio del ensayo alcanzó 364 $\mathrm{m}^{3} /$ ha (Figura $\mathrm{N}^{\circ}$ 2). Existen dos grupos principales de especies con en cuanto al volumen, uno de mayor crecimiento que comprende a E. badjensis, E. viminalis, E. globulus ssp. globulus, E. smithii, E. cypellocarpa, E. nitens, E. globulus ssp. pseudoglobulus, E. delegatensis, E. benthammii y $E$. globulus ssp. maidenii, y uno de menor crecimiento que comprende a $E$. cinerea, A. dealbata, $E$. urophylla, E. camaldulensis, A. mearnsii y C. equisetifolia.

En el caso de E. urophylla, E. camaldulensis y A. mearnsii el bajo nivel de productividad 
estuvo parcialmente asociado a una baja tasa de supervivencia, pero por otro lado, también presentan bajas tasas de crecimiento de árbol individual.

Dentro de las especies más destacadas se encuentran $E$. badjensis, con un volumen a los 14 años de $776 \mathrm{~m}^{3} / \mathrm{ha}$; E. viminalis, con $645 \mathrm{~m}^{3} / \mathrm{ha}$; y $E$. smithii, con $542 \mathrm{~m}^{3} / \mathrm{ha}$.

Al mismo tiempo, en las especies comerciales como E. globulus y E. nitens los niveles de productividad alcanzaron 610 y $493 \mathrm{~m}^{3} /$ ha, respectivamente.

Si estos valores de crecimiento son llevados a IMA (Incremento Medio Anual), $E$. badjensis alcanza a $55 \mathrm{~m}^{3} / \mathrm{ha} /$ año, E. viminalis a $46 \mathrm{~m}^{3} / \mathrm{ha} /$ año y $E$. smithii a $39 \mathrm{~m}^{3} / \mathrm{ha} /$ año.

En esta zona se planta comercialmente E. nitens con un promedio de IMA de 50 $\mathrm{m}^{3} /$ ha/año.

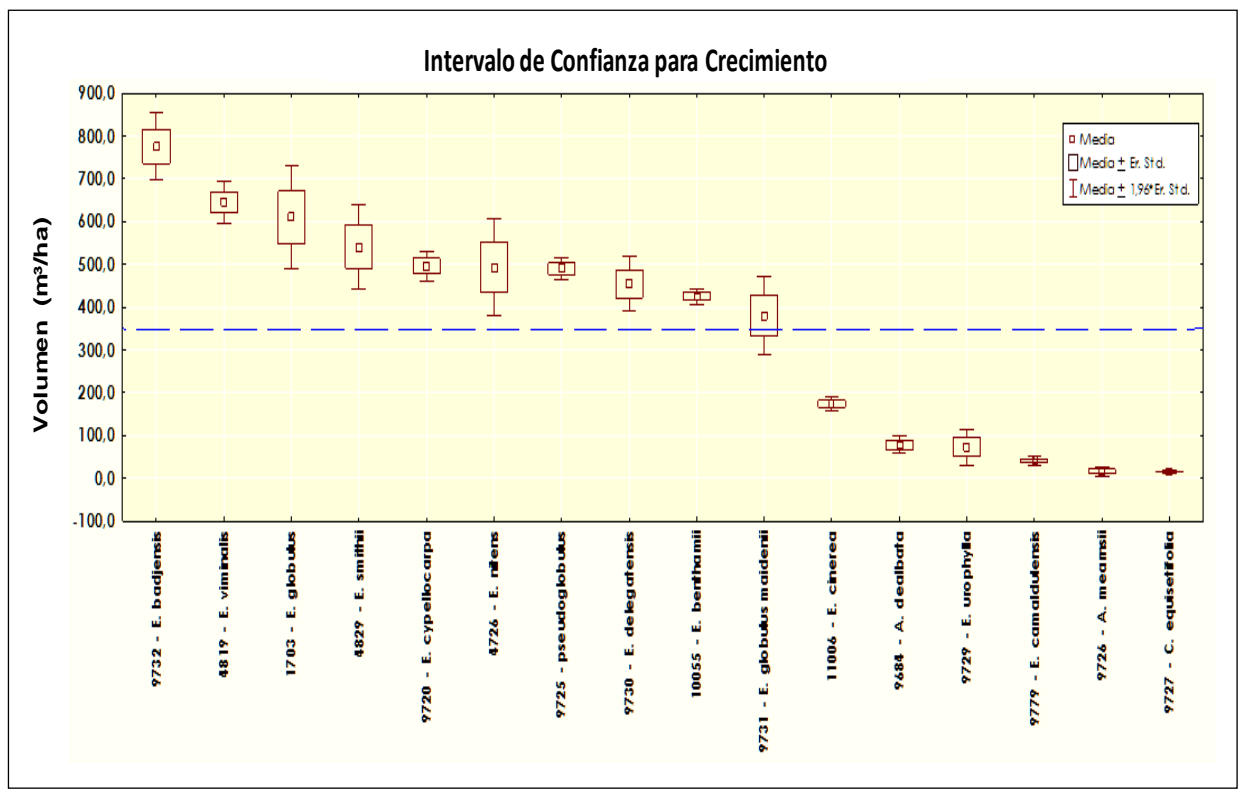

Figura $\mathrm{N}^{\circ} 2$

CRECIMIENTO DE LAS ESPECIES EN ENSAYO RUCAMANQUI 14 AÑOS EDAD

\section{Ensayo Malvén}

En el sitio de Malvén la supervivencia general del ensayo fue de $70 \%$ (Figura $\mathrm{N}^{\circ} 3$ ). Dentro de las especies de Eucalyptus la supervivencia obtenida alcanza al $72 \%$. Al igual que en el ensayo de Rucamanqui, la supervivencia es aceptable para la gran mayoría de las especies, excepto E. urophylla que fue de $26 \%$, y E. camaldulensis que fue de $41 \%$.

De igual forma que en Rucamanqui, E. urophylla y E. camaldulensis se vieron afectado por las heladas. 


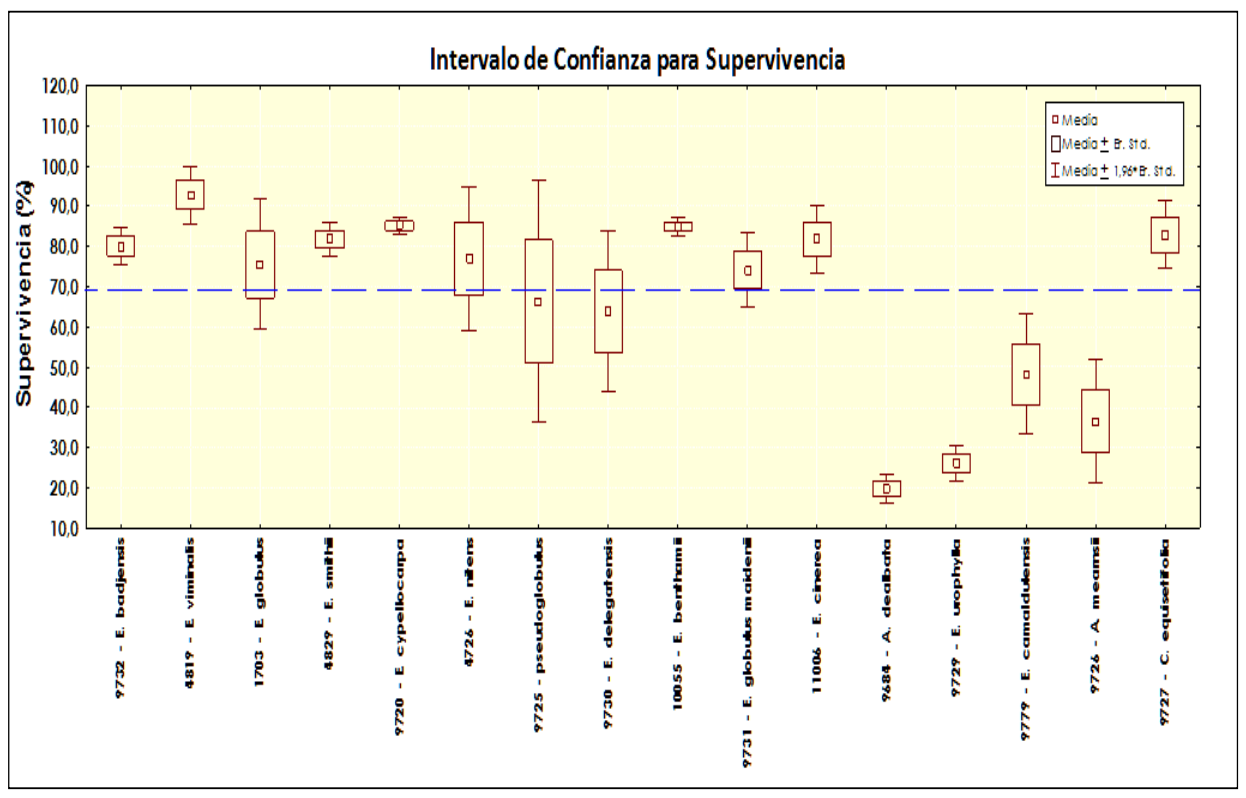

Figura $\mathrm{N}^{\circ} 3$

SUPERVIVENCIA DE LAS ESPECIES EN ENSAYO MALVÉN 14 AÑOS EDAD

Respecto al crecimiento después de 14 años, el promedio del ensayo alcanzó $427 \mathrm{~m}^{3} / \mathrm{ha}$ (Figura $\mathrm{N}^{\circ} 4$ ). Los grupos de crecimiento son similares a los del ensayo Rucamanqui, excepto que E. delegatensis y E. globulus maidenii se encuentran bajo el promedio del ensayo.

Dentro de las especies más destacadas se encuentran $E$. badjensis con un volumen a los 14 años de $887 \mathrm{~m}^{3} / \mathrm{ha}$, seguido por E. smithii con $765 \mathrm{~m}^{3} /$ ha y $E$. viminalis con $760 \mathrm{~m}^{3} / \mathrm{ha}$.

Al mismo tiempo, en las especies comerciales como E. globulus y E. nitens los niveles de productividad alcanzaron 572 y $603 \mathrm{~m}^{3} /$ ha, respectivamente.

Si estos valores de crecimiento son llevados a IMA, E. badjensis alcanza $63 \mathrm{~m}^{3} / \mathrm{ha} / \mathrm{año}, E$ smithii a $55 \mathrm{~m}^{3} / \mathrm{ha} /$ año y E. viminalis a $54 \mathrm{~m}^{3} / \mathrm{ha} /$ año.

En suelos rojo arcillosos el crecimiento anual para el caso de E. globulus alcanza 35 $\mathrm{m}^{3} /$ ha/año en plantaciones operacionales y para el caso de $E$. nitens el crecimiento alcanza en ensayos a $50 \mathrm{~m}^{3} / \mathrm{ha} /$ año.

En consecuencia, las especies destacadas parecen ser muy promisorias. 


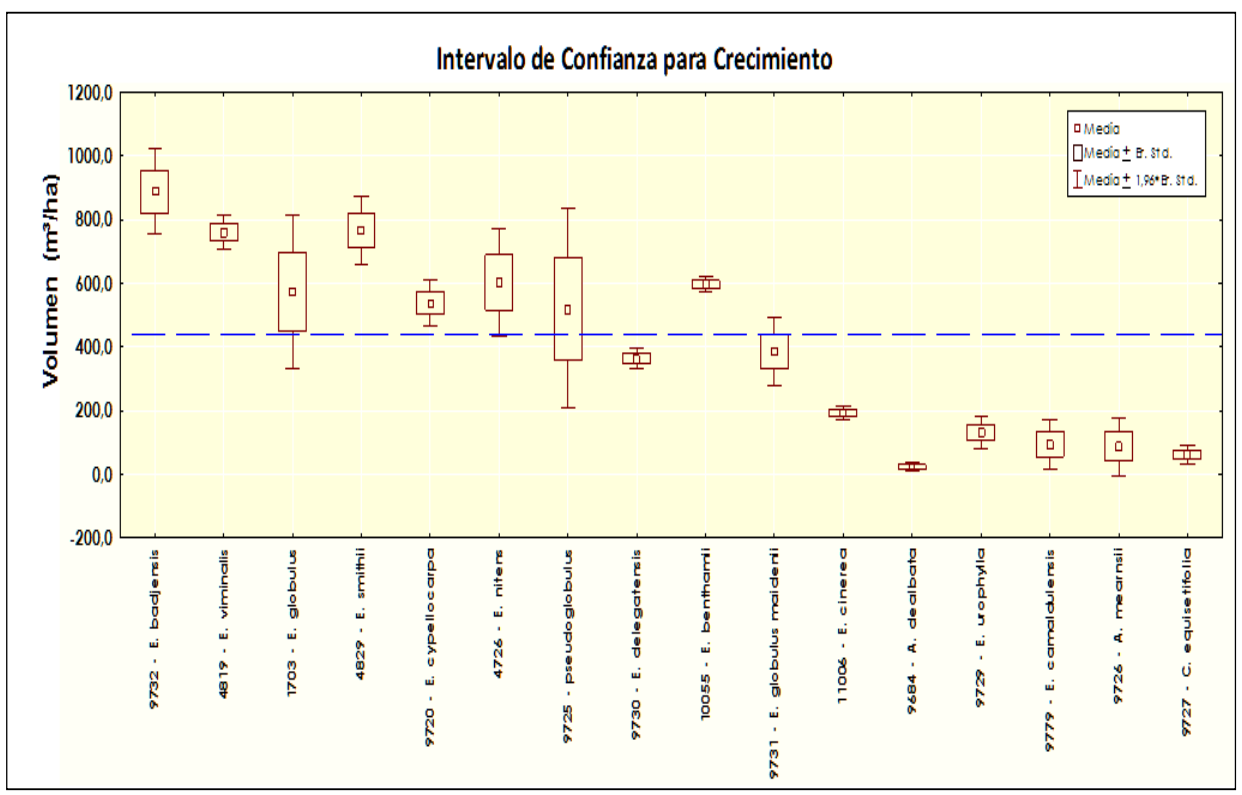

Figura $\mathrm{N}^{\circ} 4$

CRECIMIENTO DE LAS ESPECIES EN ENSAYO MALVÉN 14 AÑOS EDAD

En ensayos de especies establecidos en Australia, en el estado de Nueva Gales del Sur, se incluyeron especies como $E$. badjensis, $E$. benthamii, $E$. nitens, $E$. smithii y $E$. viminalis y se evaluó la calidad de madera después de 14 años de edad (Hicks and Clark, 2001).

La densidad básica estimada con astillas para el caso de $E$. badjensis fluctuó entre 499 y $503 \mathrm{~kg} / \mathrm{m}^{3}$, para E. smithii entre 552 y $568 \mathrm{~kg} / \mathrm{m}^{3}$, para $E$. viminalis entre 506 y $517 \mathrm{~kg} / \mathrm{m}^{3}$ y para $E$. benthamii entre 504 y $516 \mathrm{~kg} / \mathrm{m}^{3}$. Estas densidades fueron un poco inferiores a las de $E$. nitens, usado como control en estos ensayos, que fluctuó entre 546 y $553 \mathrm{~kg} / \mathrm{m}^{3}$.

También se evaluó rendimiento pulpable, variable para la cual destaca el control $E$. nitens con 50,6 - 52\%, en tanto que E. smithii registra entre $51,2-52,2 \%$; E. badjensis 48,4 $53,3 \%$ y finalmente $E$. viminalis $43,9-51,5 \%$.

En estudios anteriores de Forestal Mininco se ha muestreado E. smithii y E. viminalis en un sector de Rucamanqui y la densidad básica a los 9 años resultó de $508 \mathrm{~kg} / \mathrm{m}^{3}$ para $E$. smithii a los 14 años de $530 \mathrm{~kg} / \mathrm{m}^{3}$ para E. viminalis.

Para todas las especies probadas las semillas provienen de colectas en rodales sin mejoramiento genético, por lo que seleccionando individuos destacados y escalando en programas simples de mejora genética se pueden lograr mejores resultados que los presentados en este documento. 


\section{CONCLUSIONES}

Los resultados de estos ensayos confirman que existen otras especies promisorias que merecen dados sus crecimientos, como son los casos de E. badjensis, E. smithii y E. viminalis.

Es importante ampliar la evaluación a otras zonas del patrimonio de Forestal Mininco, como también del país para identificar así zonas en las que ests especies crecen ma's que las tradicionalmente empleadas hasta ahora.

Otro aspecto importante de abordar es la evaluación de la calidad de madera de estas especies, que ya están en edad posible de rotación, a través de estudios de su aptitud para pulpa y para dendroenergía.

Es importante avaluar también su susceptibilidad a insectos y hongos, especialmente frente al daño que produce Gonypterus sp., ya que en el periodo juvenil de este ensayo esta plaga aún no se había hecho presente..

\section{REFERENCIAS}

Boland, D., Brooker, M., Chippendale, G., Hall, N., Hyland, B., Johnstone, R., Kleinig, D. and Turner, J., 1989. Forest Trees of Australia. CSIRO, Melbourne, 687pp.

INFOR, 1986. Especies Forestales Exóticas de Interés Económico para Chile. Gerencia de Desarrollo. AF 86/32. Santiago, Chile. $168 \mathrm{p}$.

Hicks, C. and Clark, N., 2001. Pulpwood Quality of 13 Eucalypt Species. RIRDC Publication N 01/164. Barton, Australia. $38 \mathrm{p}$.

Jovanovic, T. y Booth, T., 2002. Improved species climatic profiles. RIRDC Publication $N^{\circ}$ 02/095. Barton, Australia. 68 p. 